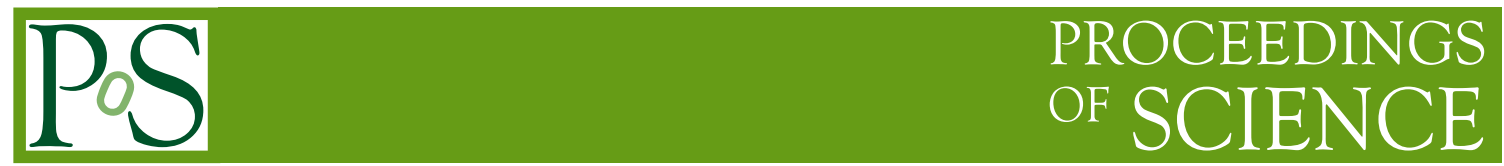

\title{
Constructive Identities for Physics
}

\author{
Andrei Rodin ${ }^{* \dagger}$ \\ Institute of Philosophy, Russian Academy of Sciences. \\ E-mail: andreiephilomatica.org
}

Homotopy Type theory instantiates a new form of axiomatic approach, which is more friendly to physics than the standard axiomatic approach stemming from Hilbert. This new axiomatic approach combines logical and geometrical methods in a new way and brings about a non-trivial constructive concept of identity applicable in various physical contexts including Quantum Mechanics and General Relativity.

Frontiers of Fundamental Physics 14 - FFP14,

15-18 July 2014

Aix Marseille University (AMU) Saint-Charles Campus, Marseille

\footnotetext{
* Speaker.

$\dagger$ The article is written with the support from RFBR, project No. 13-06-00515. I thank Vladimir Voevodsky and Noson Yanofsky for a valuable discussion
} 


\section{Axiomatization of Physics : a New Approach}

In 1900 Hilbert famously announced his list of 23 open problems for the coming century [6]; under the number 6 it included the following:

To treat by means of axioms, those physical sciences in which mathematics plays an important part.

The passed 20th century did not bring us any convincing solution of this problem. Following Schreiber [10] I would like nevertheless to point to two important developments, which may help to find such a solution in the near future. The first is Topos theory and Lawvere's idea of building physical theories synthetically within a suitable topos [7]. The second is Higher Topos theory and Homotopy Type theory (HoTT) emerged in late 2000-ies [11]. Schreiber upgrades Lawvere's axiomatic approach with the mathematical apparatus of HoTT and then uses this approach for building a tentative axiomatic Quantum Field theory.

Without trying to judge Schreiber's proposal in its more technical aspects I would like to explain here my enthusiasm about this new axiomatic approach in physics from a historical and epistemological standpoint.

Hilbert's and Lawvere's approaches to axiomatizing physics differ not only in their technical means but also in their general conceptual constitution. Hilbert describes the task of axiomatization as a "logical analysis of our intuitive capacities" (quoted after [1], p. 424 ) assuming that logical rules are fixed in advance and grounded independently. Hilbert says he derives his approach to axiomatization from Kant but in fact his approach is close to Logical Empiricism. Unlike Hilbert and Logical Empiricists Lawvere derives his conception of logic from Hegel and uses Hegel's distinction between the subjective and the objective versions of logic. While the subjective version of logic reflects only some general features of the human thinking, the objective logic, by Hegel's word, "takes the place of the former metaphysics" ([5], 21:48), which is supposed to reflect certain general features of the real world. Lawvere reconstructs Hegel's notion of objective logic mathematically in the form of internal logic of an appropriate topos, which may represent some aspect of the physical universe. In this case the axiomatization of a given theory amounts to revealing a logical semantics in the content of this theory rather than sorting out this content with some ready-made logical tools ([9], section 5.8).

In the next two Sections I expose a classical problem concerning identity of physical objects treated by Frege and then show how the Lawvere-style logical analysis empowered by HoTT provides a new solution of this problem.

\section{Identity according to Frege}

In his classical paper "On Sense and Reference" [2] Frege asks: In which precise sense the Morning Star $(M S)$ and the Evening Star $(E S)$ are said to be the same planet Venus? What particularly puzzles Frege is the following. The identity $M S=E S$ is an astronomical fact. By contrast, the assertion of identities like $M S=M S$ and $M S=$ Morning Star is trivial: these identities have no empirical content, do not depend on any astronomical theory but rather instantiate a universal logical principle and (in the second case) a mere linguistic convention. How presumably the same notion 
of identity can be used in such different senses? In order to solve this puzzle Frege developed in this paper his theory of sense and reference. Then he uses this theory for showing that assertions $M S=E S$ and $M S=M S$ has the same reference (namely the truth-value "true") but indeed different senses.

Today's reader of Frege is inclined to think of the Morning Star - Evening Star example after the pattern of Theseus Ship and other similar logical puzzles about identity. However as Frege himself remarks in the beginning of his paper he is talking here about a real scientific problem; although at the time when Frege was writing his paper this problem did no longer concern the planet Venus it did concern comets and asteroids. Taking this into consideration one may wonder why Frege's analysis lacks any discussion on observational methods used for identification of astronomical sources but instead relies upon linguistic examples having nothing to do with astronomy. The answer is that according to Frege the concept of identity is unique and applies across all possible contexts; Frege believes that this concept can and must be fixed in the pure logic prior to and independently of any mathematical or scientific theory (see for example [3], p. 74a). In this respect Frege's and Hilbert's general views on logic are similar even if they disagree about the precise sense in which logic and mathematics are said to be formal [4].

\section{Identity in HoTT and its physical interpretation}

The syntactic core of HoTT is Constructive Type theory due to Martin-Löf (MLTT) [7]. Unlike Frege Martin-Löf considers not one but two different identity concepts: the definitional identity $x=y: T$ ( where $x, y$ are terms of the same type $T$ ) and the propositional identity $\operatorname{Id}_{T}(x, y)$ : type; he assumes that the definitional identity implies the propositional one but not, generally, the other way round. The distinction between the two identities can be perfectly illustrated by Frege's $M S / E S$ example: unlike the definitional identity the propositional one admits (and epistemically talking - always requires) a proof. While in Hilbert-style deductive systems proofs are construed as metatheoretical objects (chains of formulas) in MLTT proofs are theoretical terms. Propositions and, in particular, all identity propositions like $I d_{T}(x, y)$ in MLTT are construed as types; terms of propositional types are proofs (aka witnesses) of these propositions.

Under the homotopical interpretation of MLTT types "turn into" (i.e., are modeled by) homotopy types, (i.e., by topological spaces identified up to their homotopy equivalence) while terms of these types turn into points of the corresponding spaces. Under this interpretation type $I d_{T}(x, y)$ is a space of continuous paths between points $x, y$ belonging to the given space $T$. So the proof of identity $I d_{T}(x, y)$ amounts to constructing a continuous path between these two points.

In HoTT the identity of Morning Star and Evening Star translates into the propositional identity $I d_{C}(M S, E S)$ where $C$ is an appropriately delimited type of observed celestial objects. This proposition says that two apparently different celestial objects are in fact the same. The crucial evidence in favor of this identity statement is the continuous trajectory $t$ of Venus, which connects the two different appearances of the planet (Fig. 1). Even if $t$ is a mathematically-laden theoretical construction rather than a directly observable object, it's existence can be checked empirically.

Classical kinematic principles imply that $t$, if it exists, is unique. In MLTT and HoTT this property is known as the uniqueness of identity proofs (UIP). Higher-order identities in HoTT discussed in the next Section provide a model of MLTT where UIP is false. Thus the mechanical 
interpretation of HoTT sketched above may also apply in the situation when a particle follows multiple trajectories simultaneously like in the path integral formulation of Quantum Mechanics (Fig.2).

Figure 1: Classical path

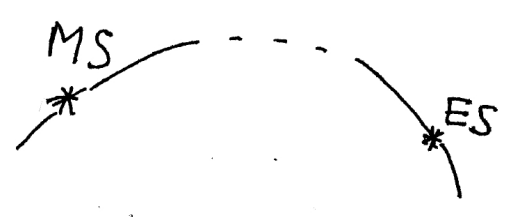

Figure 2: Quantum paths

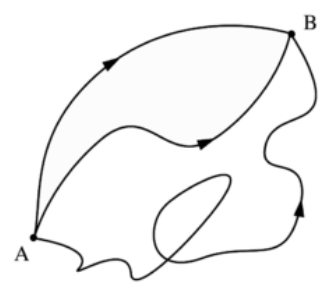

Let me stress that in the above example HoTT is not just a piece of modern mathematics successfully applied to an older astronomical theory. It is a piece of modern mathematics that supports a logical theory of identity in physics-friendly terms. This is an advantage of the new axiomatic approach, which HoTT instantiates.

\section{Higher-order identities and Objecthood}

The application of HoTT described in the last Section has been used for demonstrating a method; by itself this naive application obviously cannot have any theoretical significance. However a further feature of the identity concept in HoTT, which I describe in this Section probably can.

Let us come back to MLTT. Given two terms $x^{\prime}, y^{\prime}$ of type $I d_{T}(x, y)$, i.e. two proofs of the proposition saying that $x$ and $y$ are identical, one may form a further proposition saying that $x^{\prime}, y^{\prime}$ are the same proof, in symbols $I d_{I d_{T}(x, y)}\left(x^{\prime}, y^{\prime}\right)$. By reiterating this construction one obtains propositional types of order 3 and any higher order $n$. The "naturality" of the homotopical interpretation of MLTT amounts to the fact that Homotopy theory models this hierarchy of identity types without trivializing it. Let $x^{\prime}, y^{\prime}$ be paths with the same endpoints $x, y$. Under the homotopical interpretation of MLTT these paths model proofs of identity $\operatorname{Id}_{T}(x, y)$. Homotopy is a parametrized continuous transformation of the form $x^{\prime \prime}: x^{\prime} \rightarrow y^{\prime}$, which transforms one given path into the other. Homotopy $x^{\prime \prime}$ models proof of identity of paths $x^{\prime}, y^{\prime}$ written in symbols as $\operatorname{Id}_{I d_{T}(x, y)}\left(x^{\prime}, y^{\prime}\right)$. This latter 
Figure 3: Gravitational lensing caused by a massive body

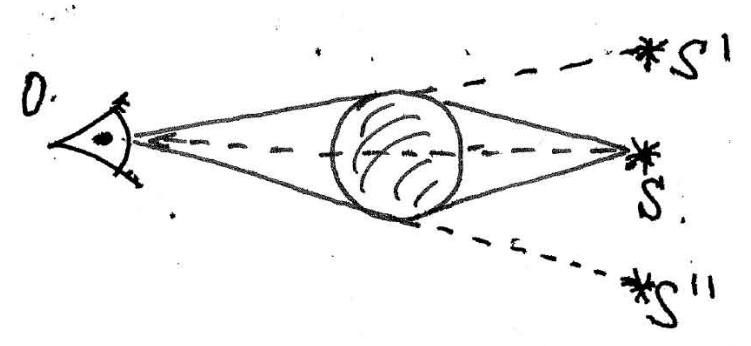

Figure 4: Gravitational lensing caused by a wormhole

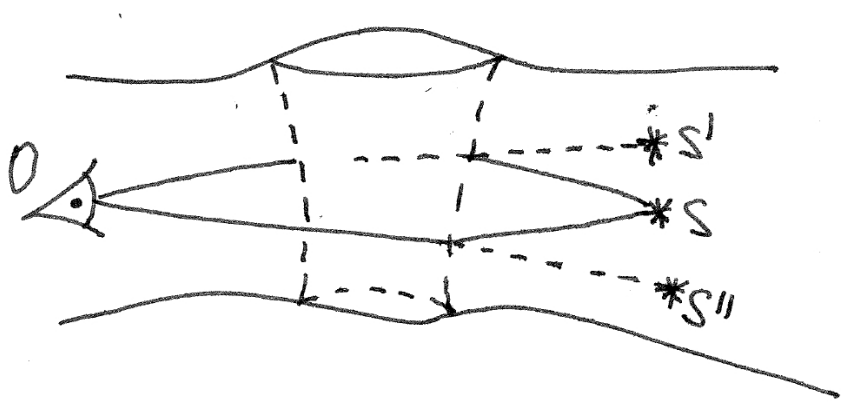

identity is modeled by the space (understood up to the homotopy equivalence of the next order) of homotopies of the form $x^{\prime \prime}$. Parametrized continuous transformations of the form $x^{\prime \prime \prime}: x^{\prime \prime} \rightarrow y^{\prime \prime}$ are homotopies of the next order, which model identities between homotopies of the previous order. This construction extends to homotopies of any order $n$ which model identities of homotopies of the previous order $n-1$.

Let us now see what kind of natural physical interpretation can be given to higher identity/homotopy types. Consider the case of gravitational lensing produced by a massive object situated between the observer $O$ and the observed source $S$ (Fig. 3). Our setting is now not the Newtonian space and time but the space-time of Einstein's General Relativity. However the situation is similar in this respect: once again we have two apparently different observable objects $S^{\prime}$ and $S^{\prime \prime}$ and want to identify them as different images of the same object $S$. The method we have used in the Venus case does not work here: there is no continuous path between $S^{\prime}, S^{\prime \prime}$. However there exists homotopy $h$, which transforms path $O S^{\prime}$ into path $O S^{\prime \prime}$. Let me assume that the existence of $h$ is empirically testable. The positive outcome of such a test can be used as an empirical evidence of identity $S^{\prime}$ and $S^{\prime \prime}$. In order to identify source $S$ we apply here the second-order identity of the form $h: I d_{I d_{C}(O, S)}\left(O S^{\prime}, O S^{\prime \prime}\right)$.

The space $H$ of homotopies of the form $h$ is topologically a disc. Thus $H$ like the path space from the Venus example is contractible, i.e., homotopy equivalent to a point. Thus we don't get here any unusual identity condition. However the situation is different when the gravitational lensing is caused by a wormhole (Fig. 4). In this case paths $O S^{\prime}, O S^{\prime \prime}$ are no longer homotopical. In order to 
identify source $S$ we shall apply the same trick at a higher dimension. Now paths $O S^{\prime}, O S^{\prime \prime}$ belong to two different path spaces, namely two discs $H^{\prime}, H^{\prime \prime}$. These discs are homotopical (through a homotopy of order 2), i.e., they are the "same" disc $H$ in the sense of the identity of order 3 . Let $d$ be a (second order) homotopy of the form $t: H^{\prime} \rightarrow H^{\prime \prime}$. The space $T$ of homotopies of this form is topologically a solid torus (not to be confused with the usual torus, which is its 2-dimensional boundary). The solid torus is homotopy equivalent to a circle but not to a point. So we get here an identity type, which is topologically non-trivial.

Even if the above examples can qualify only as toy examples they demonstrate how HoTT and the new axiomatic approach, which HoTT instantiates, allows for combining logical and geometrical methods in physics.

\section{References}

[1] L. Corry, David Hilbert and the Axiomatization of Physics (1898 - 1918), Kluwer Academic Publishers, 2004

[2] G. Frege, On Sense and Reference, in: Translations from the Philosophical Writings of Gottlob Frege, ed. by Geach and M. Black, Oxford: Basil Blackwell 1952, pp. 56-78.

[3] G. Frege, The basic laws of Arithmetic. Exposition of the System / Translated and edited, with an introduction, by M. Furth, California Press, 1964

[4] G. Frege, On the Foundations of Geometry and Formal Theories of Arithmetic, Yale University Press, 1971

[5] G.W.F. Hegel, Science of Logic, translated by George di Giovanni, Cambridge University Press, 2010

[6] D. Hilbert, Mathematical Problems, Bulletin of the American Mathematical Society, vol. 8, no. 10 (1902), pp. 437-479.

[7] W.F. Lawvere, Toposes of Laws of Motion, talk in Montreal, 1997, www.acsu.buffalo.edu/ wlawvere/ToposMotion.pdf

[8] P. Martin-Lof, Intuitionistic Type Theory (Notes by Giovanni Sambin of a series of lectures given in Padua, June 1980), Napoli: BIBLIOPOLIS, 1984

[9] A. Rodin, Axiomatic Method and Category Theory, Synthese Library vol. 364, Springer 2014

[10] U. Schreiber, Quantization via Linear homotopy types, arXiv:1402.7041

[11] V. Voevodsky, Homotopy Type Theory: Univalent Foundations of Mathematics, Institute for Advanced Study (Princeton) 2013; available at http://homotopytypetheory.org/book/ 